\title{
Throughput-Optimal Configuration of Fixed Wireless Networks
}

\author{
Aditya Karnik, Aravind Iyer, and Catherine Rosenberg, Senior Member, IEEE
}

\begin{abstract}
In this paper, we address the following two questions concerning the capacity and configuration of fixed wireless networks: (i) given a set of wireless nodes with arbitrary but fixed locations, and a set of data flows, what is the max-min achievable throughput? and (ii) how should the network be configured to achieve the optimum? We consider these questions from a networking standpoint assuming point-to-point links, and employ a rigorous physical layer model to model conflict relationships between them. Since we seek capacity results, we assume that the network is operated using an appropriate schedule of conflict-free link activations. We develop and investigate a novel optimization framework to determine the optimal throughput and configuration, i.e., flow routes, link activation schedules and physical layer parameters. Determining the optimal throughput is a computationally hard problem, in general. However, using a smart enumerative technique we obtain numerical results for several different scenarios of interest. We obtain several important insights into the structure of the optimal routes, schedules and physical layer parameters. Besides determining the achievable throughput, we believe that our optimization-based framework can also be used as a tool, for configuring scheduled wireless networks, such as those based on IEEE 802.16.
\end{abstract}

Index Terms-Capacity, fixed wireless networks, IEEE 802.16, mesh networks, optimal scheduling and routing.

\section{INTRODUCTION}

C HARACTERIZING the "capacity" of a wireless network has turned out to be a difficult problem owing to the intricacies of communication over the wireless medium. Beginning with [1], a popular approach has been to characterize the asymptotic scaling of capacity in the number of nodes (e.g., [2]). By asking for bounds only in an order sense, it has been possible to derive the trend of capacity scaling, even in the information theoretic sense [3]. However, although the knowledge of a capacity scaling law is quite valuable, it lends no insights into actual numbers for network capacity based on current technologies, or into the impact of macroscopic parameters such as transmit power budgets and modulation schemes on the network

Manuscript received August 21, 2006; revised May 17, 2007. First published March 12, 2008; current version published October 15, 2008. Approved by IEEE/ACM TRANSACTIONS ON NETWORKING Editor E. Knightly. This work was done while A. Karnik was at the University of Waterloo, Waterloo, ON, Canada, and A. Iyer was at Purdue University, West Lafayette, IN. This work was supported in part by NSERC, the National Science Foundation (NSF), and a grant from Bell University Laboratories (BUL). A short version of this paper appeared in the 44th annual Allerton Conference on Communication, Control, and Computing, September 2006.

A. Karnik and A. Iyer are with the General Motors India Science Lab, Bangalore, 560 066, India (e-mail: aditya.karnik@gm.com; aravind.iyer@gm.com).

C. Rosenberg is with the Department of Electrical and Computer Engineering, University of Waterloo, Waterloo, ON N2L 3G1, Canada (e-mail:cath@ece. uwaterloo.ca).

Digital Object Identifier 10.1109/TNET.2007.909717 capacity. These are important practical issues since wireless networks are becoming a ubiquitous part of our lives. Cellular networks already offer voice as well as data services, and IEEE 802.11 (Wi-Fi) networks are being deployed on campuses and malls creating wireless "hotspots." Wireless sensor and mesh networks are beginning to see deployment.

Even as wireless networks progress towards providing truly seamless connectivity between individuals and their environment, their commercial success hinges on their performance. Towards this end, many advanced physical layer and multiple access schemes are being incorporated into the wireless standards to extract the most out of the bandwidthand interference-limited wireless channel. For example, IEEE 802.16 (WiMAX) provides multiple burst profiles (i.e., modulation-coding schemes) which can be dynamically allocated to links by a central authority such as a base station. The standard also specifies message passing mechanisms for this centralized resource allocation (even in the "mesh mode"). Although such capabilities are available, the performance of the network depends on how well these capabilities are configured. Thus, from the point of view of network operation it is not only important to characterize the achievable network performance (capacity) but also a configuration of the advanced features achieving it. This is the problem we seek to address in this paper. Specifically, we seek answers to the following two questions.

Q1. Given a set of nodes with arbitrary but fixed locations, and a set of data flows specified as source-destination pairs, what is the maximum achievable throughput, under certain constraints on the radio parameters (e.g., regulatory constraints on transmit power)?

Q2. Further, how should the network be configured to achieve this maximum? As we explain below, by configuration, we mean the complete choice of the set of links, the flow routes, link schedules, and transmit power and modulation scheme for each link.

We approach Q1 from a networking standpoint rather than an information theoretic one to establish explicit, rather than asymptotic, analytical bounds on the capacity under currently implementable physical layer technologies. Hence, we assume point-to-point wireless links with configurable radio parameters (transmit power and modulation-coding scheme) and a specified bit-error-rate (BER) as a qualification for transmission success. Under this setting, transmission "conflicts" arise because simultaneous transmissions on arbitrarily chosen links may cause interference to one another, thereby, violating the BER requirement for some of them. Satisfaction of the BER requirement on a link can be taken to be equivalent to the condition that the signal-to-interference-and-noise ratio (SINR) at the receiver of the link remains greater than a certain threshold for the entire duration of the transmission [4]. We show that this SINR 
condition results into a conflict set for each wireless link. For a given link, the corresponding conflict set is a collection of subsets of links, such that, to meet the SINR threshold on this link, at least one link from each subset must be silent (i.e., not transmitting) when the given link is transmitting. It is clear that simultaneous transmission on links which mutually satisfy this activation constraint will all be successful, i.e., "conflict-free." Since we seek capacity results, we assume that the network is operated by means of an appropriate schedule of such conflict-free link activations. In contrast to simplistic models based on interference range or k-hop neighborhoods, our physical layer model is more involved but is based on signal decoding in the presence of noise, and is, thus, rigorous and more general (see [5]).

Our notion of the maximum throughput is the max-min flow rate, i.e., we consider maximizing the minimum end-to-end flow throughput that can be achieved in the network. This is an appropriate notion of capacity from a networking perspective, since it can represent the aggregate bandwidth demands of subscriber stations in an IEEE 802.16-like access network, or the sampling rate at which sensors produce information about their environment in a sensor network [6]. It is also the classical notion of capacity à la Gupta-Kumar [1].

Our interest in answering Q2, is two-fold. The first is to understand the structure imposed by optimality. In particular, answering Q2 will throw light on several open questions, such as whether increasing transmit power improves capacity, whether using more hops with higher data-rate links is better than using fewer low data-rate hops, and so on. Secondly, we believe that answering Q2 has direct implications for the configuration of wireless networks. This in turn has two reasons. Firstly, the notion of scheduling is not merely an artifact used to derive capacity results but in fact upcoming standards such as IEEE 802.16 provide mechanisms which enable scheduled network operation. Secondly, our model incorporates capabilities such as multiple transmit power levels, and multi-rate modulation and coding schemes, which are available for example in IEEE 802.16 , and can hence provide a complete configuration of these capabilities to achieve the optimal throughput. We shall have occasion to argue later that, in contrast to the popular dynamic approach, the machinery of assigning capacities to links by means of static link activation schedules is actually amenable to implementation. Hence, we believe it is possible, and indeed expedient, to engineer such wireless networks for optimal performance, using the techniques we develop.

We address Q1 and Q2 in a static setting, i.e., we do not model traffic and queue processes explicitly but rather work with flows. We resolve Q1 and Q2 by explicitly constructing a network that has the maximum throughput. Note that since the problem is to construct a throughput-optimal network, a topology cannot be assumed to start with, and the choice of the set of links must emerge as part of the optimal configuration. The idea, therefore, is to pose it as a problem of optimal resource allocation and routing on a "dummy network" specified by the complete directed graph on the given set of wireless nodes. Answering Q1 and Q2 is then equivalent to determining the jointly optimal flow routes, link activation schedules, and physical layer parameters which maximize the minimum flow throughput on this dummy network. Our framework can accommodate different scenarios such as one in which the transmit power and modulation scheme are to be configured and fixed or one in which nodes have the capability of employing different transmit powers and modulation schemes on their outgoing links at different instances of time. We discuss two complementary formulations dealing with the routing perspective, and the scheduling perspective.

Overall, we view our contributions as threefold.

$\mathrm{C} 1$. We develop a novel framework based on conflict sets, to characterize the maximum achievable throughput of an arbitrary fixed wireless network. This characterization generalizes the classical graph inequality, namely, that the clique number of a graph is at most equal to its chromatic number. We show that determining the maximum throughput is a computationally hard problem in general. However, we provide conditions under which a smart enumerative technique can greatly reduce the computational complexity.

$\mathrm{C} 2$. We characterize the optimal network configuration that achieves the maximum throughput. This provides several important structural insights.

- Increasing the transmit power of the nodes improves the capacity. This supports the results proved in [8], and is in contrast with power control protocols such as COMPOW [7] which recommend the use of the common minimum transmit power that guarantees connectivity.

- Scheduling constraints from conflict sets are shown to result in an extended conflict graph (say $G$ ). If $G$ is perfect, the optimal routing minimizes the transmission time through resource-constrained cliques. This implicitly implies that in the optimal configuration problem, routing and scheduling problems get separated. Thus, there is no cross-layer interaction between the two, if $G$ is perfect. If $G$ is complete, minimum medium time routing [9] is optimal. If, in addition, link data-rates are equal, minimum hop routing is optimal.

- The answer to the question whether power should be used for data-rates or range is non-trivial.

- If transmit power is not a constraint, then for a single gateway network with all traffic destined to the gateway, single hop communication between the nodes and the gateway is an optimal solution.

C3. Our framework can be used as a tool for configuring wireless (mesh) networks [10] such as those based on IEEE 802.16. We present numerical results for different scenarios of interest such as access networks with nodes deployed on a grid, with nodes placed arbitrarily, and (cellular) back-haul networks with base-stations deployed in hexagonal cells.

The rest of the paper is organized as follows. In Section II we survey the related work. The problem formulations are discussed in Section III and the numerical results are presented in Section IV. Issues related to the configuration of scheduled wireless networks are discussed in Section V. We conclude in Section VI. All the proofs are collated in Section VII.

\section{RELATED WORK}

The capacity of wireless networks in an explicit (rather than asymptotic) sense has been studied in [11], [12]. In [11], the throughput maximization problem is posed as a linear program, and lower and upper bounds to the maximum throughput are 
obtained. In comparison to [11], our results are more comprehensive since they provide important insights into the impact of radio parameters on the capacity. Moreover, instead of numerical bounds, we exploit the problem structure to solve the problem exactly. We also derive interesting analytical bounds on the maximum throughput, relating routing and scheduling. In [12] the authors present a probabilistic approach by considering a random placement of nodes. The effective throughput of a random network is modeled as a random variable and its expectation, defined as the capacity, is computed using the MonteCarlo methods. Unlike our conflict set structure, this work is built on the idea of a "schedule graph." Since we assume a given placement of nodes, our work may be seen as characterizing the capacity (and an optimal configuration) for every "sample path" of a random network.

Formulations addressing the joint optimization of routing, scheduling and power control have been explored in numerous papers (see for example, [13], [14]). In both these works, the notion of link capacity is drawn from approximations of the Shannon capacity formula. In contrast, we consider modulation schemes as determining the link data-rates. This completely changes the problem in that we have to model the conflict relationships between the wireless links.

In dynamic formulations such as [15], the authors consider the problem of dynamic routing and scheduling. The authors characterize the capacity region, and prove that a stabilizing policy is based on the celebrated differential backlog scheduling [16]. Although such a policy does not require channel and traffic statistics, it requires the knowledge on a per slot basis, of network-wide data backlogs and channel state information, in addition to the computational burden of searching through an exponential number of network configurations to see which one maximizes the differential backlog. In contrast, a static configuration such as the one we develop, is appropriate for aggregate traffic flows and has the advantage of assigning capacities to links, by means of link activation schedules which can be maintained within the framework of standards such as IEEE 802.16.

\section{PROBLEM Formulations}

In this section, we present our problem formulations in detail. Recall that we are interested in answering the questions Q1 (what is the capacity?) and Q2 (what is the optimal configuration?) given an arbitrary set of stationary wireless nodes, a set of data flows between them, and a set of available radio parameters. We address these questions in a static setting, i.e., we do not model the traffic and queue processes explicitly, and consider a configuration as fixed values of the relevant parameters, rather than as a dynamic policy responsive to traffic and/or queues. Thus, a transmission schedule is essentially seen as a (fixed) "link activation map." The assumption underlying these modeling decisions is that the traffic is static (or quasi-static [17]). This and the assumption of stationary nodes are based on realistic considerations to wireless (mesh) networks for access and back-haul, which will predominantly be fixed wireless, and will carry traffic aggregated from (many) individual users, ${ }^{1}$ and hence, should not be construed as limitations of this work.

\footnotetext{
${ }^{1}$ In the context of IEEE 802.16, the given wireless nodes would correspond to subscriber and base stations.
}

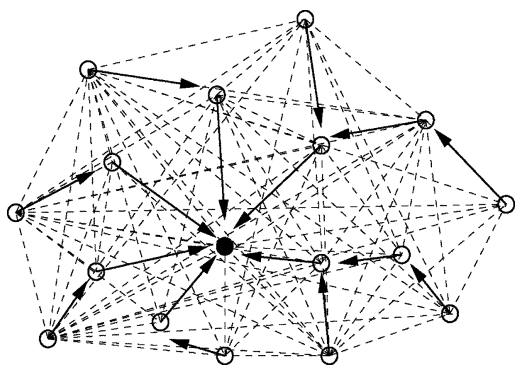

Fig. 1. An example illustrating the idea of constructing network as an optimal subgraph of the complete directed graph of wireless nodes. In this example, traffic from each node is intended for a gateway.

Seen together Q1 and Q2 are equivalent to constructing a throughput-optimal network of the given wireless nodes. The idea then is to pose this as a problem of optimal resource allocation and routing on a "dummy" network specified by the complete directed graph on the given set of wireless nodes. An optimal solution of this problem completely characterizes the capacity and a configuration achieving it; the set of links with positive transmission power allocated to them represents the selected network topology whereas the flow routes, link activation schedules and radio parameters at each link specify an optimal network configuration. Fig. 1 illustrates this idea for a set of nodes, each generating traffic intended for a gateway. The dotted lines are understood to represent directed links in both directions in the complete directed graph on the set of nodes, and the solid lines represent the optimal routes on the selected set of links.

The above resource allocation problem is intrinsically complicated owing to the interdependence of routing, scheduling and radio parameters. First, the "capacity of a link" is not only determined by its radio parameters but also by the frequency of its activation in the schedule. A schedule, however, must be conflict-free; hence a link cannot be activated arbitrarily. Moreover, with fixed radio parameters at each link, a link activation schedule results in a certain capacity for each link, which dictates the links that must be used (routing) in order to maximize throughput. At the same time, a given routing scheme specifies the total traffic flow on each link and hence dictates a link activation schedule. It thus follows that routing, scheduling and radio parameters must be jointly optimized in order to maximize the network throughput. We investigate two equivalent formulations of this joint optimization problem. The reason being that the capacity characterization and the answers to the structural questions posed in Section I can be obtained from the first formulation, (TO), with considerable ease, whereas the second formulation, (TOLP), highlights the scheduling perspective, and, being a linear program, can be used as an efficient computational tool to obtain the numerical results. First, we state the modeling assumptions.

\section{A. Assumptions and the Model}

Some basic notation is in order. $N$ denotes the number of given static wireless nodes; their set is denoted by $\mathcal{N}$. Each node will be identified with its location. $\mathcal{L}$ denotes the set of all possible links among these nodes. Transmission power constraints may imply that certain links are infeasible, in which case their data-rates under the associated radio configuration are taken to be 0 (the notion of "infeasibility" is formalized in the following discussion). 
Hence, $\mathcal{L}$ may be taken as the set of links in the complete directed graph on $N$ nodes without loss of generality. The cardinality of $\mathcal{L}$ is denoted by $L$. Links are assumed to be directed; $l \in \mathcal{L}$ is also represented as $\left(l_{o}, l_{d}\right)$, where $l_{o}$ and $l_{d}$ denote the originating and the destination nodes respectively. $\mathcal{L}_{i}^{O}$ (resp. $\mathcal{L}_{i}^{I}$ ) denotes the set of links outgoing from node $i$ (resp. incoming to $i$ ).

Radio Parameters and PHY Layer: In this non-informationtheoretic setup, communication errors cannot be completely eliminated; hence "success" is in the sense of achieving a specified bit error rate (BER). We, therefore, assume a given fixed BER requirement. We also assume that all nodes transmit on a common frequency band.

Let $P_{l}, l \in \mathcal{L}$ denote the transmission power on link $l$. $\mathbf{P}:=$ $\left(P_{1}, \ldots, P_{L}\right)$ denotes the power vector. $z_{l}$ denotes the modulation-coding scheme used on link $l ; \mathbf{z}:=\left(z_{1}, \ldots, z_{L}\right) . \mathcal{Z}$ denotes the set of available modulation and coding schemes. It is assumed that $\mathcal{Z}$ is bounded and the transmit power on each link is constrained. ${ }^{2}$ Thus, $\mathcal{P}$ denotes the set of allowable power vectors; for example, $\mathcal{P}=\left\{\mathbf{P} \mid 0 \leq P_{l} \leq \bar{P}, l=1, \ldots, L\right\}$, for some $\bar{P}$ depending on regulatory constraints. Since our main interest is in the data-rate provided by a modulation and coding scheme, the scheme will be identified with the bits per symbol it can represent; ${ }^{3}$ hence with slight abuse of notation, $z_{l} \in R_{+}$ and it denotes the bits per symbol provided by the corresponding modulation and coding scheme.

Under $\mathbf{P}$, the SINR on link $l, \gamma_{l}$, is given by $G_{l l} P_{l} /\left(N_{0}+\right.$ $\left.\sum_{l^{\prime}} G_{l^{\prime} l} P_{l^{\prime}}\right)$ where the summation in the denominator is over the links transmitting simultaneously with $l . N_{0}$ denotes the (thermal) noise power, and $G_{l l}$ (resp. $G_{l^{\prime} l}$ ) denotes the channel gain on link $l$ (resp. from link $l^{\prime}$ to $l$ where it is understood that it refers to the gain from $l_{o}^{\prime}$ to $l_{d}$ ). Recall that channel gains typically constitute path loss, shadowing and fading components. In this paper, we assume that $G_{\{.\}}$s are time-invariant and known. The BER specification translates into a minimum SINR requirement (or SINR threshold), $\beta\left(z_{l}\right)$ for each $z_{l}$ on link $l$ [4]; note that, for narrow-band systems $\beta\left(z_{l}\right)>1$. This essentially means that a transmission on link $l$ is considered to be successful if $\gamma_{l}$ is at least $\beta\left(z_{l}\right)$ for the duration of the transmission. ${ }^{4}$ Given $\mathbf{P}$, let $\mathcal{Z}_{l}\left(P_{l}\right) \subseteq \mathcal{Z}$ denote the set of modulation-coding schemes which can achieve the BER specification in the absence of co-channel interference on link $l$; it is clear that under $\mathbf{P}$ only schemes in $\mathcal{Z}_{l}\left(P_{l}\right)$ can be used. A link is said to be infeasible (and assigned data-rate of 0$)$ if $\mathcal{Z}_{l}\left(\tilde{P}_{l}\right)$ is empty. $\mathcal{Z}(\mathbf{P}):=\Pi \mathcal{Z}_{l}\left(P_{l}\right)$.

Remark: The assumption of a common frequency band has been made to keep the exposition simple. It is straightforward to include multiple frequency channels in our framework through the notion of artificial links introduced in Section III-C. In view of recent studies which have shown that fixed wireless channels are relatively static in urban-suburban areas [18], our assumption of time-invariant and known channel gains is not unreasonable. Moreover, the channel characteristics can be mea-

\footnotetext{
${ }^{2}$ These constraints reflect the limitations on implementing very high rate modulation schemes, and regulatory restrictions imposed on transmit power in various bands of the frequency spectrum.

${ }^{3}$ Two modulation-coding schemes may have the same data-rate, but may require different minimum SINRs to satisfy the given BER requirement. We ignore this issue in this paper but it can be incorporated easily in our framework.

${ }^{4}$ This condition is in general more restrictive than the BER requirement. By considering them equivalent we are being conservative.
}

sured from the estimation and feedback procedures available in wireless standards. Here the radio parameters at each link are assumed to take values from real numbers. For transmission power, this is a natural assumption. In view of numerous modulation-coding schemes yielding a wide variety of data-rates, this is not an unreasonable assumption for modulations as well. A more practical scenario of discrete power levels and finite modulation schemes can be handled by simple specialization of our formulations as discussed in Section III-C and Section III-D. $\square$

Link Conflict Sets: Given $\mathbf{P} \in \mathcal{P}$ and $\mathbf{z} \in \mathcal{Z}(\mathbf{P})$, the condition for a successful transmission on link $l$, viz., $\gamma_{l} \geq \beta\left(z_{l}\right)$, results into a "conflict set" $\mathcal{D}_{l}(\mathbf{z}, \mathbf{P})$ for the link $l$. Each $D \in$ $\mathcal{D}_{l}(\mathbf{z}, \mathbf{P})$ is a subset of $\mathcal{L}$ with the interpretation that if all the links in $D$ are transmitting simultaneously with link $l$ (using transmission powers given by the power vector $\mathbf{P}$ ) then the transmission on link $l$ fails. This can be seen as follows. Let $\mathbf{v}$ be an $L$-dimensional $\{0,1\}$ vector and let $\mathcal{V}_{l}=\left\{\mathbf{v}: G_{l l} P_{l} /\left(N_{0}+\right.\right.$ $\left.\left.\sum_{l^{\prime}} G_{l^{\prime} l} P_{l^{\prime}} v_{l^{\prime}}\right)<\beta\left(z_{l}\right)\right\}$. Then each $D \in \mathcal{D}_{l}(\mathbf{z}, \mathbf{P})$ corresponds to a $\mathbf{v} \in \mathcal{V}_{l}$ that cannot be represented as (modulo 1) addition of any other vectors in $\mathcal{V}_{l} ; l^{\prime} \in D$ if $v_{l^{\prime}}=1$. Therefore, to guarantee a successful transmission on link $l$, at least one link from each $D \in \mathcal{D}_{l}(\mathbf{z}, \mathbf{P})$ must be silent.

Remark: It is clear that under a realistic physical layer model, conflicts among links may be more complicated than those representable by k-hop neighborhoods for each link or by the protocol model (also see [5]). Moreover, the conflict relationships may not even be "binary," to be represented by a conflict graph. Note that only in the special case when all the $\mathcal{D}_{l}$ 's defined above consist of singleton subsets $D$, can the conflict sets be represented as a conflict graph. Interestingly, however, the conflict sets can be seen as specifying multiple conflict graphs; in each of these graphs, a vertex corresponds to a link in the network, and for each link $l$ one interferer is selected from each $D \in \mathcal{D}_{l}(\mathbf{z}, \mathbf{P})$ and an edge established, thus satisfying the requirement that "at least one link from $D$ must be silent." Note that the conflict structure intricately depends on node locations.

Link Scheduling and Capacity: We assume that transmissions are co-ordinated (possibly, though not necessarily, by a central controller) through activation schedules and not by a distributed medium access control (MAC) protocol. Let $\mathcal{S}$ denote the power set of $\mathcal{L}$; cardinality of $\mathcal{S}$ is denoted by $S$. Sets in $\mathcal{S}$ will be arbitrarily indexed $1, \ldots, S$. A transmission schedule is an $\mathrm{S}$-dimensional vector $\alpha:=\left(\alpha_{1}, \alpha_{2}, \ldots, \alpha_{S}\right)$ such that $\alpha_{i} \geq 0, i=1$, $\ldots, S$ and $\sum_{i=1}^{S} \alpha_{i}=1 . \alpha$ can be interpreted in two ways. First, suppose that time is divided into "slots" and that transmissions can be initiated only at the slot boundaries for the duration of a slot. $\alpha$ then denotes the marginal distribution of a stationary-ergodic scheduling process. Second, assuming data to be fluid, the length of a transmission can be taken to be infinitely divisible. Then defining a frame as an interval of unit length to be repeated infinitely, $\alpha$ denotes a "time allocation map" for each frame; $\alpha_{i}$ is the fraction of time links in the $i$ th set transmit simultaneously in a frame. This interpretation is of interest in IEEE 802.16 since the standard allows protocol data units (PDUs) to be fragmented at a fine scale, and provides a frame structure to allocate fractions of time to the links in the network.

It is clear from our physical channel model that for fixed $\mathbf{P}$ and $\mathbf{z}$, transmission on link $l$ in an activation set $A \in \mathcal{S}$ will 
be successful only if $l^{\prime} \in A$ are such that at least one link from each $D \in \mathcal{D}_{l}(\mathbf{z}, \mathbf{P})$ is not in $A$. If this condition holds for each $l \in A$, we call $A$ an "independent set" for the conflict structure imposed by $\mathcal{D}_{l}(\mathbf{z}, \mathbf{P})$ 's. Recalling the fact that $\mathcal{D}_{l}(\mathbf{z}, \mathbf{P})$ 's realize multiple conflict graphs, an independent set $A$ is a graph-theoretic independent set in one of those graphs. Given $\mathbf{P}$ and $\mathbf{z}$, denote by $\mathcal{I}(\mathbf{z}, \mathbf{P})$ the set of independent sets in $\mathcal{S}$ and by $\mathcal{A}(\mathbf{z}, \mathbf{P})$ the set of non-conflicting schedules, i.e., $\left\{\alpha \mid \alpha_{i} \geq 0, i \in \mathcal{I}(\mathbf{z}, \mathbf{P}), \alpha_{i}=0, i \notin \mathcal{I}(\mathbf{z}, \mathbf{P}), \sum_{i=1}^{S} \alpha_{i}=1\right\}$.

For each $\alpha \in \mathcal{A}(\mathbf{z}, \mathbf{P})$, the maximum rate of data transmission of link $l$, referred to as its capacity and denoted by $c_{l}\left(z_{l}, \alpha\right)$, is a well-defined quantity equaling the product of data rate $c_{l} z_{l}$ and the rate of successful activation $\sum_{i \in \mathcal{I}_{l}(\mathbf{z}, \mathbf{P})} \alpha_{i} . c_{l}$ denotes the number of channel accesses per unit time (determined by the channel bandwidth [4]) and $\mathcal{I}_{l}(\mathbf{z}, \mathbf{P})$ denotes the set of independent sets which contain $l$.

Flows and Routing: Data transfer requirements are specified in terms of $M$ flows, the set of which is denoted by $\mathcal{F}$. Each flow $f \in \mathcal{F}$ is identified with a source-destination pair $\left(f_{s}, f_{d}\right) ; f_{s}$, $f_{d} \in \mathcal{N} . \mathcal{R}_{f}$ is the set of all possible routes $f$ can be routed on. Each route $r \in R_{f}$ is specified by a sequence of links $\left[l^{1}, \ldots, l^{j}\right]$ for some $j$, such that $l_{o}^{1}=f_{s}, l_{d}^{j}=f_{d}, l_{d}^{i}=l_{o}^{i+1}$ for $i=$ $1, \ldots, j-1$, and the directed graph induced by $\left[l^{1}, \ldots, l^{j}\right]$ does not contain any cycles. $\lambda_{f}$ denotes the flow rate of $f . \phi_{f}^{r}$ denotes the fraction of traffic of flow $f$ routed on $r \in \mathcal{R}_{f}$. Clearly $\phi_{f}^{r} \geq$ 0 and $\sum_{r \in R_{f}} \phi_{f}^{r}=1 . \mathcal{R}_{f}^{l}$ denotes the set of routes of $f$ going over link $l$.

\section{B. Formulation I: Routing Perspective}

The following is the throughput optimization problem which we refer to as (TO).

$$
\begin{aligned}
& \sum_{f \in \mathcal{F}} \lambda_{f}\left(\sum_{r \in \mathcal{R}_{f}^{l}} \phi_{f}^{r}\right) \leq c_{l}\left(z_{l}, \alpha\right) \quad l=1, \ldots, L \\
& \sum_{r \in \mathcal{R}_{f}} \phi_{f}^{r}=1, \phi_{f}^{r} \geq 0 \quad f=1, \ldots, M \\
& \lambda \leq \lambda_{f} \quad f=1, \ldots, M \\
& \lambda \geq 0, \alpha \in \mathcal{A}(\mathbf{z}, \mathbf{P}), \mathbf{z} \in \mathcal{Z}(\mathbf{P}), \mathbf{P} \in \mathcal{P} .
\end{aligned}
$$

Proposition 3.1: An optimal solution exists for (TO).

Proof: See Section VII.

Having settled existence, we show that the answer to the question whether capacity improves with scaling transmit power at each node is affirmative. Let $\lambda(\mathbf{z}, \mathbf{P})$ denote an optimal solution of (TO) for fixed $\mathbf{z}$ and $\mathbf{P}$. For any vector $\mathbf{y}:=\left(y_{1}, \ldots, y_{k}\right)$, $\xi \mathbf{y}:=\left(\xi y_{1}, \ldots, \xi y_{k}\right)$ for $\xi \in R$. Then:

Proposition 3.2: For $\xi \geq 1, \lambda(\mathbf{z}, \mathbf{P}) \leq \lambda(\mathbf{z}, \xi \mathbf{P})$.

Remark: This result may seem slightly surprising, since increasing the transmit power also increases the interference. However, the effect of scaling transmit power (while fixing the modulation rate) on each link is twofold. First, the SINR on each link that is feasible under the initial transmit power improves implying that with power scaling an independent set of these link remains independent and a non-independent set may become independent. Second, certain links, which were infeasible initially, may become feasible, thereby, increasing the number of feasible links among nodes. As a consequence of these two effects, the set of independent sets (and hence the set of non-conflicting schedules) is non-decreasing as the transmit power is scaled.

Proof of Proposition 3.2: See Section VII.

Corollary 3.1: If all the nodes must use the same transmit power, then $(\bar{P}, \ldots, \bar{P})$ achieves the maximum in (TO) for any fixed $\mathbf{z}$.

The questions of whether power should be used for improving range or data-rate and whether using more hops with higher data-rates, is better than using fewer lower data rate hops, are not so straightforward. Informally the reason is as follows. Assume that $\mathbf{P}$ is fixed and that all nodes must use the same modulation scheme, $z$. Then the data rate $c_{l} z$ increases with $z$, however, $\mathcal{A}\left(z_{1}, \mathbf{P}\right) \subseteq \mathcal{A}\left(z_{2}, \mathbf{P}\right)$ for $z_{1} \geq z_{2}$. Thus, a higher modulation scheme increases the data-rate but may also reduce the set of non-conflicting schedules. Hence, even if all nodes must employ a common transmit power, the highest possible modulation scheme for that power need not achieve capacity. The following subsection tries to take a unified perspective of the above questions, by investigating the structure of the optimal routes.

\section{Structure of the Optimal Routes}

In order to throw some light on the issues raised above, we investigate the properties of optimal routing for fixed $\mathbf{z}$ and $\mathbf{P}$. With an understanding that these parameters are now fixed, we do not explicitly refer to them. In such a case (TO) takes the following form. We refer to this problem as (TOFINITE).

$$
\begin{array}{cc}
\max \lambda & \\
\sum_{f \in \mathcal{F}} \lambda_{f}\left(\sum_{r \in \mathcal{R}_{f}^{l}} \phi_{f}^{r}\right) \leq c_{l} \sum_{i \in \mathcal{I}_{l}} \alpha_{i} & l=1, \ldots, L \\
\sum_{r \in \mathcal{R}_{f}} \phi_{f}^{r}=1, \phi_{f}^{r} \geq 0 & f=1, \ldots, M \\
\lambda \leq \lambda_{f} & f=1, \ldots, M \\
\lambda \geq 0, \alpha \in \mathcal{A} &
\end{array}
$$

where $\mathcal{A}$ denotes the (fixed) set of non-conflicting schedules of all links and $c_{l}$, with slight abuse of notation, denotes the datarate determined by bit-clocking and modulation rate. Observe that $\mathcal{A}$ is compact and convex.

(TOFINITE) is important for two reasons. First, the "structural properties" of optimal routing (and scheduling) required to answer the above questions can be inferred from it since it is posed for any values of $\mathbf{z}$ and $\mathbf{P}$, including the optimal ones. Moreover, it can be used to characterize an optimal solution of (TO) as $\max _{\mathbf{z} \in \mathcal{Z}(\mathbf{P}), \mathbf{P} \in \mathcal{P}} \lambda(\mathbf{z}, \mathbf{P})$ through the Maximum theorem. Second, (TO) reduces to (TOFINITE) in the case of discrete power levels and finite modulation schemes, a case that is of direct relevance in the context of practical wireless networks. ${ }^{5}$ Basically, if at each link the transmission power $P_{l}$ must be chosen from given discrete levels and $Z_{l}$ from some finite set of modulation-coding schemes, then (TO) can be cast simply as an optimal routing problem. The idea is to replace a link

\footnotetext{
${ }^{5}$ The name (TOFINITE) is used to reflect the finiteness of radio parameters.
} 
between nodes $i$ and $j$ by multiple "artificial links," each one corresponding to a feasible combination of transmit power and modulation. ${ }^{6}$ Thus, optimal selection of power and modulation is translated into optimal selection of "links." Since all the feasible links are, thus, given a priori, the conflict structure is fixed, and $\mathcal{A}$ corresponds to the set of non-conflicting schedules of all "artificial" links.

Proposition 3.3: An optimal solution exists for (TOFINITE). Proof: See Section VII.

For ease of notation, we do not make the dependence of either fixed $\mathbf{P}$ and $\mathbf{z}$, or discrete $\mathbf{P}$ and $\mathbf{z}$ on the optimal solution of (TOFINITE) explicit, but simply denote it by $\lambda^{*}$.

We now investigate (TOFINITE) as a parametric optimization problem to answer the questions pertaining to the interplay of range and data-rate in the choice of links in an optimal configuration, as posed above. Let $\phi:=\left(\phi_{f}^{r}\right)_{f \in \mathcal{F}, r \in \mathcal{R}_{f}}$, $\Phi=\left\{\phi \mid \sum_{r \in \mathcal{R}_{f}} \phi_{f}^{r}=1, \phi_{f}^{r} \geq 0, f \in \mathcal{F}, r \in \mathcal{R}_{f}\right\}$, and $\bar{\lambda}:=\left(\lambda_{f}\right)_{f \in \mathcal{F}}$. Consider now a parameterized optimization problem (TOPARAM) with parameter $\phi$, i.e.,

$$
\max _{\Theta(\phi)} \lambda
$$

where $\Theta(\phi):=\left\{(\lambda, \bar{\lambda}, \alpha) \mid \sum_{f \in \mathcal{F}} \lambda_{f}\left(\sum_{r \in \mathcal{R}_{f}^{l}} \phi_{f}^{r}\right) \leq\right.$ $c_{l} \sum_{i \in \mathcal{I}_{l}} \alpha_{i}, l=1, \ldots, L, \lambda \leq \lambda_{f}, f=1, \ldots, M, \lambda \geq$ $0, \alpha \in \mathcal{A}$ \}. Here, $\Theta(\phi)$ represents the constraint set for a fixed routing $\phi$, i.e., the set of all link schedules and flow throughputs that are feasible under $\phi$. Denote by $\left(\lambda^{*}(\phi), \bar{\lambda}^{*}(\phi), \alpha^{*}(\phi)\right)$ an optimal solution of (TOPARAM).

Proposition 3.4:

1) An optimal solution exists for (TOPARAM) for each $\phi$.

2) $\lambda^{*}=\max _{\phi \in \Phi} \lambda^{*}(\phi)$.

3) Lagrange multipliers exist for (TOPARAM).

Remark: The problem (TOPARAM) can be seen as separating the problem of optimal scheduling from the problem of optimal routing, via route parameterization. Proposition 3.4 justifies this separation, since the optimal solution $\lambda^{*}$ of (TOFINITE) can be achieved through optimal scheduling, once the optimal routes have been selected and fixed.

Proof of Proposition 3.4: See Section VII.

Remark: Using Proposition 3.4 (3), the optimality conditions of (TOPARAM) yield the following.

$$
\begin{gathered}
\sum_{f \in \mathcal{F}} \tau_{f}^{*}=1 \\
\left(\lambda_{f}^{*}(\phi)-\lambda_{f}\right)\left(\tau_{f}^{*}-\sum_{l \in \mathcal{L}} \pi_{l}^{*}\left(\sum_{r \in R_{f}^{l}} \phi_{f}^{r}\right)\right) \geq 0 \\
\alpha^{*}(\phi) \in \arg \max _{\mathcal{A}} \sum_{l \in \mathcal{L}} \pi_{l}^{*} c_{l} \sum_{i \in \mathcal{I}_{l}} \alpha_{i}
\end{gathered}
$$

where, $\tau_{f}^{*}$ (resp. $\pi_{l}^{*}$ ) denotes the flow (resp. link) Lagrange multiplier. Clearly, setting $\lambda_{f}=\lambda^{*}(\phi)$ for all $f \in F$ also results in an optimal solution of (TOPARAM). (4) implies that this is

\footnotetext{
${ }^{6}$ For uniformity of notation, we continue to denote by $\mathcal{L}$ (resp. $L$ ) the set of all artificial links (resp. cardinality of $\mathcal{L}$ ). Links will be assumed to be arbitrarily numbered. The definitions of link activation schedule and flow route continue to hold (see Section III-A).
}

the unique solution if at least one link on the routes of each flow $f$ is bottlenecked (i.e., flow equals its capacity). Interpreting $\pi_{l}^{*}$ as the price of link $l$, (5) implies that an optimal schedule maximizes the revenue from link activation; observe that independent set $i$ fetches a revenue of $\sum_{l \in i} \pi_{l}^{*} c_{l}$.

First let us assume that the conflict structure is specified by a single conflict graph $G$. Later we will show how conflict set based scheduling constraints can be represented on a single conflict graph. Let vertex $l$ in graph $G$ be assigned a weight equal to $\sum_{f \in \mathcal{F}} \sum_{r \in \mathcal{R}_{f}^{l}} \phi_{f}^{r} / c_{l}$ for given $\phi \in \Phi$. Let $\mathcal{C}$ be set of cliques in $G$ with $w_{c}(\phi)$ denoting the weight of clique $c \in \mathcal{C}$; by weight of a clique we mean the sum of the weights of vertices in that clique. Thus, $w_{c}(\phi)=\sum_{l \in c}\left(\sum_{f \in \mathcal{F}} \sum_{r \in \mathcal{R}_{f}^{l}} \phi_{f}^{r} / c_{l}\right)$.

Proposition 3.5: For some $\kappa \in(0,1]$ which depends on the conflict graph $G$

$$
\frac{\kappa}{\min _{\phi} \max _{c} w_{c}(\phi)} \leq \lambda^{*} \leq \frac{1}{\min _{\phi} \max _{c} w_{c}(\phi)} .
$$

Proof: See Section VII.

Remark: If $G$ is perfect, $\kappa=1$, hence, $\lambda^{*}(\phi)=$ $1 / \max _{c} w_{c}(\phi)$. For the case when the conflict graphs are modeled as unit disk graphs $\kappa=0.46$ [22].

Let $\hat{\phi} \in \arg \min _{\phi \in \Phi} \max _{c \in C} w_{c}(\phi)$. Then Proposition 3.5 can equivalently be stated as

Proposition 3.6: For some $\kappa \in(0,1]$ which depends on the conflict graph $G$,

$$
\kappa \lambda^{*} \leq \lambda^{*}(\hat{\phi}) \leq \lambda^{*}
$$

Proof: See Section VII.

Remark: Proposition 3.5 and Proposition 3.6 characterize the capacity purely in terms of a routing problem $\left(\min _{\phi} \max _{c} w_{c}(\phi)\right)$. Further, the solution of this problem will result in a throughput within a constant factor of the optimal. $\square$

In general, the conflict structure specified in terms of the conflict sets, is not necessarily representable by a single conflict graph. So we take the following approach to arrive at Proposition 3.5 in a general setting. The idea is to "embed" multiple conflict graphs specified by the conflict sets in a larger conflict graph. This is done by considering multiple copies of each link, each copy basically realizing one combination of activation constraints given by its conflict set. Recall that multiple combinations of these constraints give rise to multiple conflict graphs. For example, for link $m$ let $\mathcal{D}_{m}=\left\{\left\{l_{1}\right\},\left\{l_{2}, l_{4}\right\},\left\{l_{3}, l_{5}\right\}\right\}$. Then the conflict graph is constructed by replacing link $m$ by a clique of size 4 , with copies of link $m$ as vertices, say, $m^{1}, m^{2}, m^{3}$ and $m^{4}$, with edges to $\left\{l_{1}, l_{2}, l_{3}\right\},\left\{l_{1}, l_{4}, l_{3}\right\},\left\{l_{1}, l_{2}, l_{5}\right\},\left\{l_{1}, l_{4}, l_{5}\right\}$ respectively. In general, if $\mathcal{D}_{m}=\left\{D_{1}, D_{2}, \ldots, D_{k}\right\}$, then link $m$ would be replaced by a clique of size $\left|D_{1}\right| \times\left|D_{2}\right| \times \ldots \times\left|D_{k}\right|$. Since the links $l_{1}, \ldots, l_{5}$ themselves may have similar copies, by an edge between say $m^{1}$ and $l_{5}$, we mean edges from $m^{1}$ to all the "virtual" copies of $l_{5}$. Of course, every $m^{i}$ has an edge to every $m^{j}, i, j=1,2,3,4, i \neq j$ in the conflict graph, to represent the fact that only one of these copies may be activated at a time. Again using the idea of "artificial" links, in the "extended" network, link $m$ is now replaced by its virtual copies $m^{1}, \ldots, m^{4}$. By appropriately redefining the routing 
variables $\phi$, the optimization problem over this extended network and the corresponding conflict graph has the same form as (TOFINITE). Since the independent sets as constrained by the conflict sets, and the graph-theoretic independent sets on the extended conflict graph are identical, the following holds.

Proposition 3.7: (TOFINITE) over the given conflict sets and (TOFINITE) over the extended network and conflict graph are equivalent.

Thus, Proposition 3.5 and Proposition 3.6 also hold in a general setting, but on an extended conflict graph. Motivated by this result, let us consider the routing problem $\min _{\phi} \max _{c \in \mathcal{C}} w_{c}$. The problem can equivalently be posed as:

$$
\begin{gathered}
\sum_{l \in c} \frac{\sum_{f \in \mathcal{F}} \sum_{r \in \mathcal{R}_{f}^{l}} \phi_{f}^{r}}{c_{l}} \leq w \quad c \in \mathcal{C} \\
\sum_{r \in \mathcal{R}_{f}} \phi_{f}^{r}=1, \phi_{f}^{r} \geq 0 \quad f=1, \ldots, M \\
w \geq 0 .
\end{gathered}
$$

Certainly, from the remark made earlier, there is a clear motivation to study problem (7) for perfect graphs, as it directly provides the optimal routing we sought to obtain via (TOFINITE). In general, due to Proposition 3.6, the formulation in (7) is guaranteed to result in a throughput which is within a constant factor of the optimal (in the sense of (TOFINITE)).

Let $\mu_{c}^{*}$ (resp. $\nu_{f}^{*}$ ) denote the Lagrange multiplier associated with the $c$ th clique constraint (resp. the $f$ th routing constraint). Then the Karush-Kuhn-Tucker conditions for (7) imply the following.

$$
\begin{gathered}
\sum_{c \in \mathcal{C}} \mu_{c}^{*}=1 \\
\left(\phi_{f}^{r *}-\phi_{f}^{r}\right)\left(\nu_{f}^{*}-\sum_{c \in \mathcal{C}} \mu_{c}^{*}\left(\sum_{l \in c_{r}} \frac{1}{c_{l}}\right)\right) \geq 0
\end{gathered}
$$

where $c_{r}$ is the set of links in clique $c$ used by route $r$. Note that $\mu_{c}^{*} \geq 0$ since in (7) the equality constraint $\left(\sum_{r \in \mathcal{R}_{f}} \phi_{f}^{r}=\right.$ 1) may equivalently be replaced by the inequality constraint $\left(\sum_{r \in \mathcal{R}_{f}} \phi_{f}^{r} \geq 1\right)$. Therefore, interpreting $\mu_{c}^{*}$ as the "price" clique $c$ sets for airtime on its links and $\nu_{f}$ the price flow $f$ is willing to pay, (9) implies that flow $f$ chooses that route which minimizes its total expenditure, where the expenditure in clique $c$ equals $\mu_{c}^{*}\left(\sum_{l \in c}\left(1 / c_{l}\right)\right)$; note that since $c_{l}$ is the data rate on $l, 1 / c_{l}$ is the time to send one unit of data. Thus, the optimal route minimizes the total cost of air-time in the resource-constrained cliques it passes through. Moreover, if $\phi_{f}^{r *}>0$ for more than one $r \in R_{f}$ then the expenditure in each of them must be equal. An interesting case is when $c_{l}=1$ (normalized) for all links. Then $\mu_{c}^{*}\left(\sum_{l \in c}\left(1 / c_{l}\right)\right)=\mu_{c}^{*} m_{c_{r}}$, where by $m_{c_{r}}$ we denote the number of links in clique $c$ used by route $r$. Thus, in this case, the optimal routes employ the minimum hop path through cliques which have positive prices. Our result is a generalization of an obvious result that if the conflict graph $G$ is such that only one link can transmit at a time (i.e., it is complete), then the minimum medium time routing [9] is optimal. In the case of a complete conflict graph, if all the links have the same data-rate, then minimum hop routing is optimal. Note that, by definition so called "single cell" networks have complete conflict graphs.

We now recall the questions posed earlier, namely, should power be used for range or data-rate and is using more hops with higher data-rates better than using fewer lower data rate hops? In the light of the above development, it is clear that their answers depend intricately on the cliques in the conflict structure. As a partial characterization, we can say that if the (extended) conflict graph for any fixed $\mathbf{z}$ and $\mathbf{P}$ is perfect, then in an optimal configuration many short but higher data-rate links will be used if the sum of air-times, i.e., $1 / c_{l}$ through a resource-constrained clique is lesser than that of fewer lower data-rate links. Thus, whether power will be used for range or data-rate is not determinable for links individually; power will be used for range at a node if the corresponding route can traverse the constrained clique in lower air-time. We will illustrate this interplay through the numerical examples in Section IV.

\section{Formulation II: Scheduling Perspective}

$$
\begin{aligned}
& \max \lambda \\
& \sum_{l \in \mathcal{L}_{i}^{o}} x_{l}^{f}-\sum_{l \in \mathcal{L}_{i}^{I}} x_{l}^{f}= \begin{cases}0 & i \notin\left\{f_{s}, f_{d}\right\} \\
\lambda_{f} & i=f_{s} \\
-\lambda_{f} & i=f_{d}\end{cases} \\
& i=1, \ldots, N, f=1, \ldots, M \\
& \sum_{f \in \mathcal{F}} x_{l}^{f} \leq c_{l} \sum_{k \in I_{l}} \alpha_{k} \quad l=1, \ldots, L \\
& \sum_{k \in \mathcal{I}} \alpha_{k}=1 \\
& 0 \leq \lambda \leq \lambda_{f} \quad f=1, \ldots, M .
\end{aligned}
$$

While the formulation emphasizing the scheduling aspect can be done in full generality as (TO), here we discuss only a specialization similar to (TOFINITE). Therefore, it corresponds to two distinct scenarios-(i) fixed $\mathbf{z}$ and $\mathbf{P}$, and (ii) $z_{l}$ and $P_{l}$ taking finite discrete values for each link.

Let $x_{l}^{f}$ denote the flow rate of $f \in \mathcal{F}$ on link $l$. Then throughput optimization can be cast as a linear program (TOLP) in (10). The equivalence of (TOLP) and (TOFINITE) is direct. Therefore, we denote an optimal solution of (TOLP) by $\lambda^{*}$ as well. An interesting characterization of $\lambda^{*}$ can be obtained from the dual of (TOLP) as follows.

Proposition 3.8:

$$
\lambda^{*}=\min _{V} \max _{i \in \mathcal{I}} \sum_{l \in i} \nu_{l} c_{l}
$$

where the set of dual variables $V=\left\{(\mu, \nu, u) \in R^{N M} \times\right.$ $R_{+}^{L} \times R_{+}^{M} \mid \sum_{f \in \mathcal{F}} u_{f}=1, u_{f} \leq \mu_{f_{s}, f}-\mu_{f_{d}, f}, f \in \mathcal{F} ;$ and $\left.\mu_{l_{o}, f}-\mu_{l_{d}, f} \leq \nu_{l}, f \in \mathcal{F}, l \in \mathcal{L}\right\}$.

It can be shown that for $i, j \in \mathcal{I}$ with $\alpha_{i}^{*}>0$ and $\alpha_{j}^{*}>0$, $\sum_{l \in i} \nu_{l}^{*} c_{l}=\sum_{l \in j} \nu_{l}^{*} c_{l}$. Thus, if $\nu_{l}$ is interpreted as the cost of using link $l$, then the cost of using the total data-rate of every actively used independent set is equalized.

Remark: Note that a similar interpretation applies from the Lagrangian of (TOPARAM) as well. Recalling (5), it is clear that for a given routing $\phi$, if $\alpha_{i}^{*}(\phi)>0$ and $\alpha_{j}^{*}(\phi)>0$, then the revenue generated by the independent sets must be equal, i.e., $\sum_{l \in i} \pi_{l}^{*} c_{l}=\sum_{l \in j} \pi_{l}^{*} c_{l}$. 


\section{E. Generalizing a Classical Graph Inequality}

Besides giving an interesting complementary characterization of $\lambda^{*}$ in terms of the flow routes and cliques in $G$, and link schedules and independent sets in $G,(6)$ and (11) lead to an interesting result. Combining (6) and (11) we get

$$
\min _{V} \max _{i \in \mathcal{I}} \sum_{l \in i} \nu_{l} c_{l}=\lambda^{*} \leq \frac{1}{\min _{\phi} \max _{c} w_{c}(\phi)}
$$

where the equality holds if $G$ is perfect.

Now consider the situation when the flows are chosen in such a way that the only feasible links are those between each sourcedestination pair (thus, each flow is essentially one-hop). If the link data-rates are all identical, and the link activation constraints result in singleton conflict sets for each link, then $\lambda^{*}$ is nothing but $1 / \gamma(G)$ where $\gamma(G)$ is the chromatic number of the graph $G$. In that case, we also have that $\min _{\phi} \max _{c} w_{c}(\phi)=\omega(G)$, where $\omega(G)$ is the clique number of $G$. In other words, (12) reduces to $\omega(G) \leq \gamma(G)$, i.e., the clique number of $G$ is less than or equal to the chromatic number; equality holds if $G$ is perfect. Thus, (12) captures a generalization of the classical graph inequality.

\section{NUMERICAL RESULTS}

In this section, we present several numerical results of interest by solving (TOLP). We start with a hardness result, and then describe the technique we use to address the computational complexity.

\section{A. Addressing Computational Complexity}

The problem of computing the optimum throughput, and the optimal configuration is computationally hard in general. This is a consequence of the following hardness result.

Proposition 4.1: The problem of determining the max-min throughput of a network, given any conflict structure specified in terms of the conflict sets, is NP-hard.

Remark: In [11], the authors consider a related problem of determining the maximum throughput of a single data flow in a wireless network, with the conflict structure specified in terms of a single conflict graph. This related problem is proved to be NP-hard, via a reduction of the maximum independent set problem to their problem.

Proof of Proposition 4.1: The proposition immediately follows by observing, that any instance of the problem considered in [11] is also an instance of our problem.

In order to solve (TOLP) numerically, we need to construct non-conflicting schedules. To devise non-conflicting schedules, we need independent sets in the conflict structure (see Section III-A). Although this is a computationally hard problem in general, we use a smart enumerative technique to compile the independent sets for several cases of interest. For this technique, we make the following additional assumptions.

A1. The channel gains are modeled by isotropic path loss, i.e., for each $l, l^{\prime} \in \mathcal{L}, G_{l l^{\prime}}=\left(|x-y| / d_{0}\right)^{-\eta} ; x$ and $y$ denote the locations of $l_{o}$ and $l_{d}^{\prime}$ resp. $\eta$ is the path loss exponent and $d_{0}$ is the far-field crossover distance.

A2. All nodes are physically separated by at least a distance $d_{\min }$.

A3. The network is confined to a bounded area in space, say a square of size $L \times L$.
Remark: In A1 we do not take into account the location-dependent shadowing component of the channel gain. We feel this is reasonable for two reasons. First, the shadowing component is relatively static and not time-varying [18]. Secondly, our interest is more in observing overall trends, and distilling structural properties, rather than predicting exact values. A2 is necessary since $d^{-\eta}$ grows unbounded as $d$ approaches zero, thereby yielding arbitrarily high channel gains.

Recall that we operate under a given fixed BER specification. For this BER, denote the lowest SINR threshold, i.e., the one corresponding to the lowest rate modulation scheme, by $\beta_{\min }$.

Proposition 4.2: Under the assumptions A1-A3, the maximum size of an independent set (or the maximum number of links that can be scheduled simultaneously) is bounded above by a constant $B$ which depends only on $\eta, d_{\min }, L$ and $\beta_{\min }$.

Proof: See Section VII.

It is clear from Proposition 4.2 that we need to enumerate only those subsets of links that are of a size smaller than the bound, and check whether those subsets are independent sets. Once the independent sets have been so enumerated, solving the problem is just a matter of solving the linear program (10). In order to get an idea of the extent of complexity reduction this technique can result in, consider some concrete numbers pertaining to the $5 \times 5$ grid in Fig. 6 , in which there are a total of 86 potential links for the stated physical layer parameters. The set of all subsets of links has, thus, cardinality $2^{86}$. The size of the maximum independent set turns out to be bounded by 6 . The set of all subsets of links of size 6 or less, has cardinality of the order of $86^{6}$. Of all these subsets, the actual number of independent sets is only 1451, which puts (TOLP) well within the prowess of "off-the-shelf" linear program solvers.

Before we move on to the numerical results, we summarize the physical layer parameters used to derive them. Three modulation schemes are considered with normalized data-rates of 1, 4 and 8, and SINR thresholds of $10 \mathrm{~dB}, 20 \mathrm{~dB}$ and $25 \mathrm{~dB}$, respectively. ${ }^{7}$ All the nodes use omni-directional antenna, and operate using the same set of transmit power levels and the same set of modulation and coding schemes. $\eta$ and $d_{0}$ are taken to be 4 and $0.1 \mathrm{~m}$ resp. $N_{0}$ equals $-100 \mathrm{dBm}$. In what follows, the term "transmission range" (for a given power and modulation scheme) would be used to refer to the maximum transmitter-receiver separation under which successful packet decoding remains possible for that power and modulation in the absence of any co-channel interference. We report normalized throughput values since the exact numbers as such may not be of practical interest.

\section{B. Two Flows: Interference-Avoiding Routing}

We start with an example of two data flows on a $5 \times 5$ grid (see Fig. 2). The "red" flow (denoted by red straight lines) originates at the bottom left corner node, and is destined to the node immediately left of the top right corner node. The "blue" flow (shown using blue curved lines) originates at the node immediately right of the bottom left corner node, and is destined to the top right corner node. All the other nodes merely act as relays. The unit grid separation (distance between adjacent nodes along the grid-side) is taken to be $8 \mathrm{~m}$. All the nodes use a transmit power

\footnotetext{
${ }^{7}$ These are typical values of an uncoded QAM (Quadrature Amplitude Mod-
} ulation) system for a BER of $10^{-6}$. 


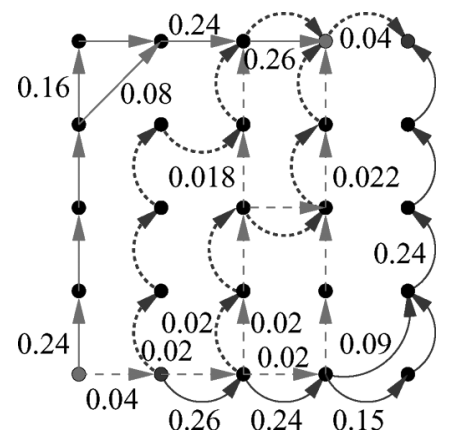

Fig. 2. Two Flows on a $5 \times 5$ grid: Modulation 1 , Transmit Power $-7 \mathrm{dBm}$.

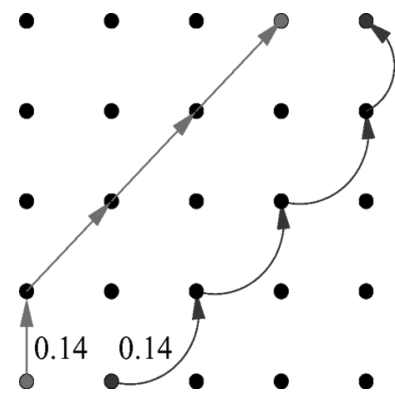

Fig. 3. Minimum hop routes for the two Flows in Fig. 2.

of $-7 \mathrm{dBm}$, and use modulation 1 . This results in a transmission range of $11.9 \mathrm{~m}$, which is slightly greater than the unit grid diagonal (distance between adjacent nodes along the diagonal). Fig. 2 depicts the routes and normalized capacities of the different links, under the optimal configuration. On the optimal routes, indicated in Fig. 2, the dotted links carry less than $10 \%$ of the total traffic and more than $90 \%$ of the total traffic is carried along the periphery over the solid links; the routes use only two diagonal links in spite of having a transmission range greater than the unit grid diagonal. It is clear that the optimal routing is far from minimum hop, and multiple routes are used for the two sources. Some data is routed along common paths and links, however, the bulk of the data is routed so that the flows "avoid" each other. This is an illustration of "interference-avoiding" routing, a phenomenon which we shall notice in upcoming examples as well.

If the minimum hop routing is imposed on the two flows, specifically using the routes shown in Fig. 3 , the (normalized) throughput is $1 / 7$, only half of $2 / 7$ which can be achieved using the optimal configuration (Fig. 2). Thus, the blind application of minimum hop or minimum medium time routing [9] could result in significant throughput degradation.

\section{Wireless Access Network on a Grid}

Our next study investigates the achievable throughput of a network deployed on a grid. We assume that all the nodes (e.g., subscriber stations in IEEE 802.16) generate the same amount of traffic intended for the sink/gateway node (e.g., base station in IEEE 802.16) at the bottom left corner (which generates no traffic). This provides us with a regular topology that is simple yet reasonable to study access networks. The separation between adjacent nodes along the grid side is $8 \mathrm{~m}$.

1) Single Power and Modulation Level: In this scenario, we consider a $5 \times 5$ grid and assume that all the nodes use one common transmit power and modulation scheme. The optimal

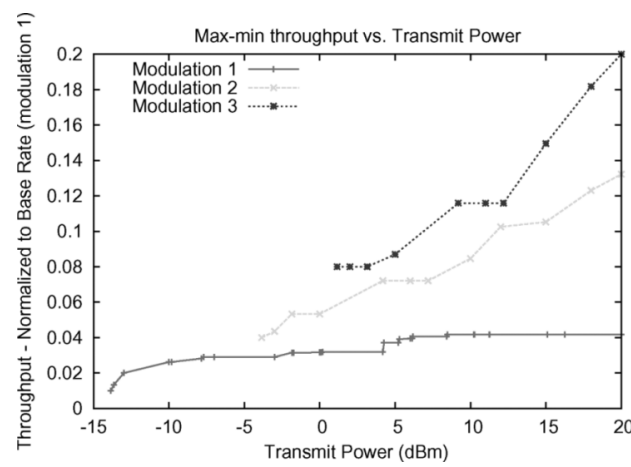

Fig. 4. Variation of $\lambda^{*}$ with Transmit Power (in $\mathrm{dBm}$ ).

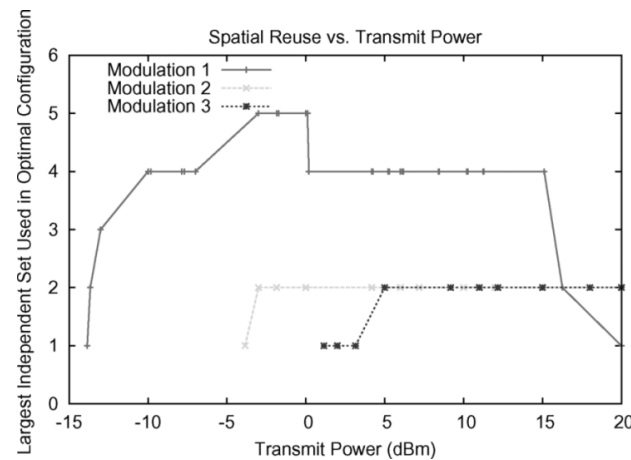

Fig. 5. Variation of Spatial Reuse with Transmit Power (in dBm).

max-min throughput is plotted as a function of the transmit power in Fig. 4 for different modulation and coding schemes. The leftmost point on each of the three sets of curves indicates the minimum transmit power at which the network is connected. The lower rate modulation schemes provide connectivity at low transmit powers, however, cannot obtain any significant gains in throughput at higher transmit powers. From Fig. 4, it is clear that for this scenario, for any value of the transmit power, the highest rate modulation scheme under which the network is connected should be used. However, as argued in Section III-C, this need not be the case in general.

For modulation 1, at the transmit power level of $20 \mathrm{dBm}$, the network operates as a single-hop network, with each node directly communicating with the gateway. Although throughput is non-decreasing with transmit power, clearly there would be no improvement in the throughput, using modulation 1 , if the power is increased beyond $20 \mathrm{dBm}$. For modulation 2 and modulation 3 , this point would correspond to some higher transmit power level, not shown in Fig. 4. As can be seen from Fig. 4, about $50 \%$ or $85 \%$ of the maximum throughput for modulation 1 , is achieved at much lower powers $(-13 \mathrm{dBm}$ and $0 \mathrm{dBm}$, respectively). The same behavior is expected for modulation 2 and modulation 3 as well, if transmit power levels higher than $20 \mathrm{dBm}$, are considered.

To better understand the reason behind the initial steep increase and gradual flattening out of the throughput curves, let us look at Fig. 5 which shows the size of the largest independent set used in the optimal configuration (which is a measure of spatial reuse) as a function of the transmit power. Focusing on the curve for modulation 1 , we can observe that when the network is just barely connected, all the links formed have little immunity 


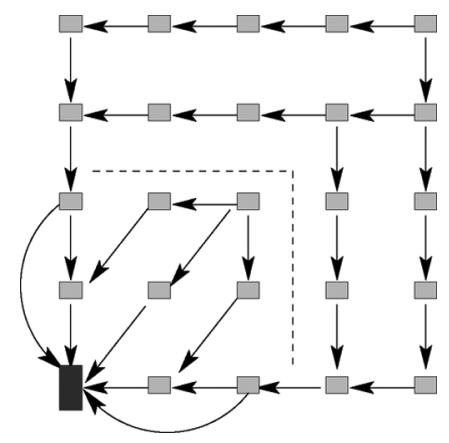

Fig. 6. $5 \times 5$ grid: Modulation 1, Transmit Power $-1.85 \mathrm{dBm}$.

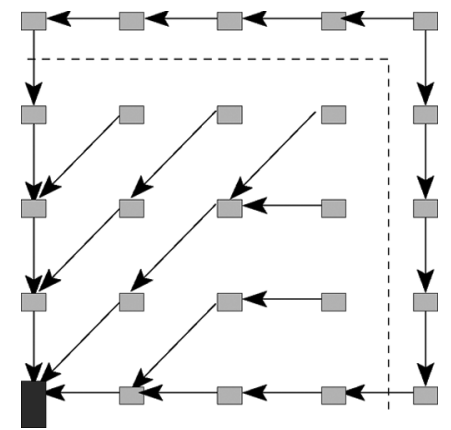

Fig. 7. $5 \times 5$ grid: Modulation 2, Transmit Power $4.185 \mathrm{dBm}$.

to interference. Hence, they can only be scheduled one at a time (conflict graph is complete). As the transmit power increases, the spatial reuse in the network steadily improves as the links become more and more immune to interference. Beyond a certain transmit power, as longer links start getting created, it becomes more favorable for the data to be routed over longer links which means fewer hops at the cost of spatial reuse. Here the corresponding increase in throughput is not as dramatic. Also, observe that using higher rate modulation schemes means less immunity to interference and reduced spatial reuse, although in this scenario, it is more than made up by the data-rate increase.

Fig. 6 and Fig. 7 depict the optimal routing under different choices of transmit power and modulation and coding scheme. The range of a node in the configuration in Fig. 6 is $16 \mathrm{~m}$ which is twice the unit grid side, and in Fig. 7 it is $12.7 \mathrm{~m}$ which is more than the unit grid diagonal. Observe that, in both cases the optimal routes are not minimum hop for every node. Within the region indicated by the dotted line in both the figures, the nodes use minimum hop paths, although in Fig. 6 some nodes split their data along multiple paths not all of which are minimum hop. Also, observe that the nodes along the diagonal, beyond the dotted line, route their data along the periphery of the network. This is another illustration of interference-avoiding routing.

2) Multiple Power and Modulation Levels: We now consider an example in which 2 transmit power levels and 2 modulation and coding schemes are available at each node. The topology we consider is a $4 \times 4$ grid. Fig. 8 shows the variation of the max-min throughput with transmit power under different capabilities. Here the term " 2 Modulations" refers to the fact that the two modulation schemes, modulation 1 and modulation 2 (as described earlier) are both available at all the nodes. Similarly, the term " 2 Power levels" refers to the fact that all the nodes

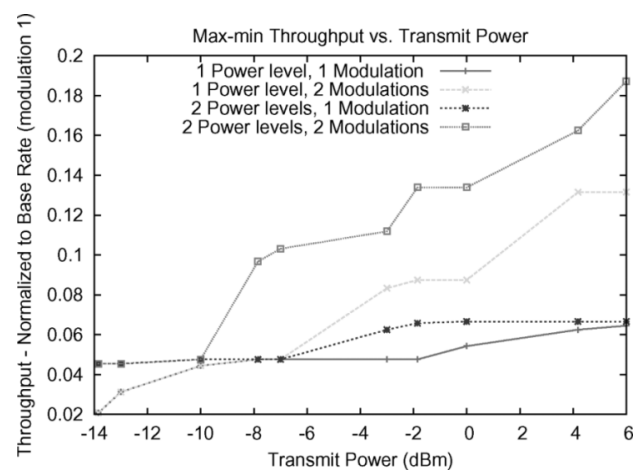

Fig. 8. Variation of $\lambda^{*}$ with Transmit Power (in $\mathrm{dBm}$ ).

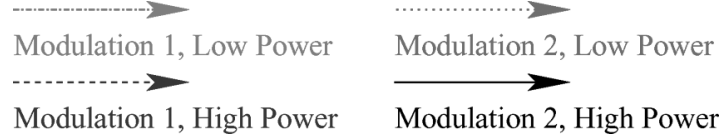

Fig. 9. Legend for Figs. 10, 11, 12, and 13.

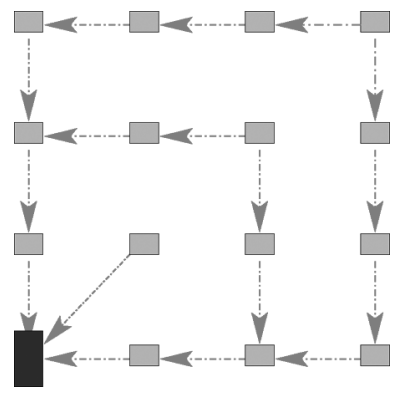

Fig. 10. $4 \times 4$ grid: Modulation 1 , Transmit power $=-3 \mathrm{dBm}$.

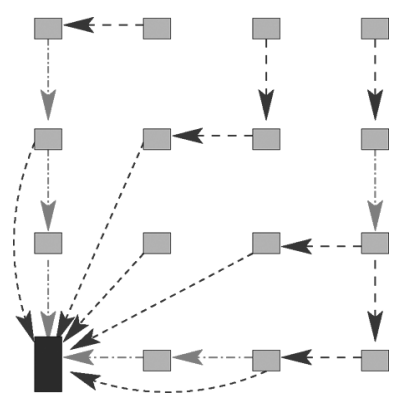

Fig. 11. $4 \times 4$ grid. Modulation 1 . Transmit power $=-3 \mathrm{dBm}$ and $2 \mathrm{dBm}$.

can operate at two power levels, viz., a low power level which is marked on the $\mathrm{x}$-axis, and a high power level which is $5 \mathrm{dBm}$ higher. Note that one cannot directly compare the throughput curves for the "1 Power level" cases with those corresponding to " 2 Power levels" because of the availability of a higher power level; they are all represented on the same graph merely for convenience. The same qualitative behavior that we observed in Section IV-C, can be observed here again. In this case, since the lower rate modulation scheme is always available, connectivity is ensured under all scenarios for the range of powers considered. Particularly, the two curves corresponding to " 2 Modulations," have the benefits of connectivity at lower power levels and high throughput at higher power levels, in contrast with the curves in Fig. 4.

Fig. 9 provides the legend used to depict links in the optimal configuration in Figs. 10, 11, 12, and 13. Fig. 10 depicts the 


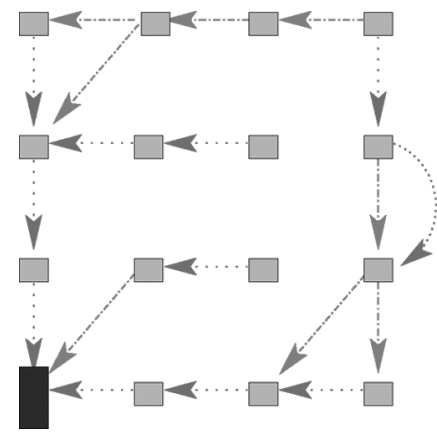

Fig. 12. $4 \times 4$ grid. Modulation 1 and modulation 2. Transmit power $=$ $-3 \mathrm{dBm}$.

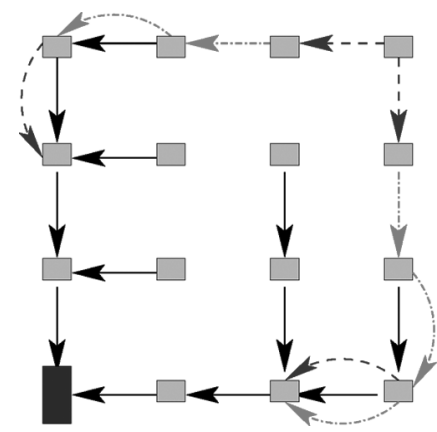

Fig. 13. $4 \times 4$ grid. Modulation 1 and modulation 2. Transmit power $=$ $-3 \mathrm{dBm}$ and $2 \mathrm{dBm}$.

optimal configuration with one power level $(-3 \mathrm{dBm})$ and one modulation scheme (modulation 1). It just confirms what we have seen so far: minimum hop routing in the vicinity of the sink; and interference-avoiding routing elsewhere. The interesting point is to compare Fig. 10 to Fig. 11 in which the ability to use a second higher power level $(2 \mathrm{dBm})$ has been added. As indicated in Fig. 9, here the magenta (dot-dash) links represent the links using $-3 \mathrm{dBm}$ power, and the blue (dash) links use $2 \mathrm{dBm}$ power (all links use modulation 1). Due to the availability of higher power links, more minimum hop paths are used in the vicinity of the sink. Since there is no data-rate improvement in using blue (dash) links, they are used solely to increase range. Now, let us compare Fig. 10 with Fig. 12 in which the ability to additionally use modulation 2 , has been added. Here the red (dot) links represent the modulation 2 links operating at $-3 \mathrm{dBm}$. Since the red (dot) links offer considerable data-rate improvement they are extensively used in the network. However, the magenta (dot-dash) links are still used far away from the sink, owing to the lower data volumes and higher immunity to interference.

Note that there is no simple rule to explain these optimal routes. However, all these observations appear to be in accordance with the interpretation that the optimal routes tend to minimize the transmission times through resource-constrained cliques. Thus, by using lower data-rate (and therefore more robust) links far away from the sink, these do not become part of the bottleneck clique around the sink. This is confirmed in Fig. 13 which shows the optimal configuration when both power levels and modulation schemes can be used. The black (solid) links are the high power, high data-rate links. Rather than using long blue (dash) links as in Fig. 11, the nodes in the center use short black (solid) links since the higher data-rate they offer, offsets the number of hops required, in terms of minimizing the transmission time. As we argued in Section III-B, the optimal routing is intricately connected with how cliques are created in the (extended) conflict graph, and in general, it is an interesting balance of links of different physical layer parameters.

\section{Practical Issues: Configuration OF WIRELESS NETWORKS}

Although current wireless technologies may not have datarates comparable to their wired counterparts such as DSL, they hold the key to remove the "last mile" bottleneck by connecting customers, even in remote places, possibly with faster deployment at lower costs. Hence for a service provider a wireless (mesh) network for access or back-haul is an attractive proposition. That such a network should be optimized for performance is obvious: the infrastructure cost incurred by the service provider must be offset by the profit from charging customers for specific Internet services. However, our numerical results supported by analytical results show that an optimal configuration is a delicate balance of the network and the physical layer parameters, and hence that it cannot be arrived at by simple scenario analysis. Naïve configurations, on the other hand, can result in significant throughput degradation. We, therefore, argue that in deploying such networks, in particular those based in IEEE 802.16, an offline design approach based on our optimization framework is a compelling choice.

Firstly, our formulation models the physical layer rigorously (also see [5]) and, as discussed in Section III, it is geared towards wireless access/back-haul networks by considering aggregate traffic flows and arbitrarily placed nodes. It also encompasses numerous generalizations, for example, weighted max-min objective to yield throughputs proportional to per node traffic demands, and directional antennas at the PHY layer [23]. Secondly, the linear form of (TOLP) means that an efficient computational tool can be built to devise optimal configurations. Moreover, note that the central source of complexity in arriving at an optimal configuration is enumeration of independent sets (see TOLP). In view of (mostly) time-invariant channel gains [18], such enumeration needs to be done only once (or very infrequently). This, along with the fact that TOLP is a linear program, means that optimal configurations can be determined efficiently whenever traffic requirements change, for example, dependent on time-of-day. Finally, our approach can work very well with IEEE 802.16 since the transmission schedules and physical layer parameters (burst profiles) can be maintained within the message passing framework of IEEE 802.16.

The offline design does not mean that the network is designed offline and manually configured. The process of configuration can be automated. The only "offline" aspect is that the capacity is assigned via static schedules to cater to projected (aggregate) traffic requirements. Note that we do not claim to have addressed all the engineering issues involved in such an approach, but we merely advocate static configuration of wireless networks as a viable possibility.

As an example, consider first the problem of constructing an optimal access network of 23 subscriber stations and two basestations (gateways) placed arbitrarily. Nodes 5 and 9 in Fig. 14 


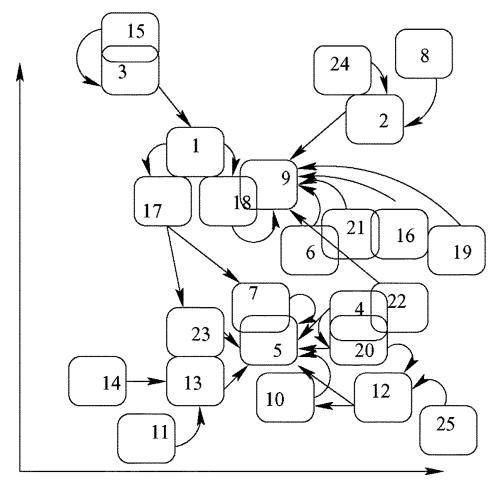

Fig. 14. Optimal access network of arbitrarily placed subscriber stations. Nodes 9 and 5 are base stations. Transmit power is $-7.7 \mathrm{dBm}$. Up-link rate $\lambda^{*}=0.065$.

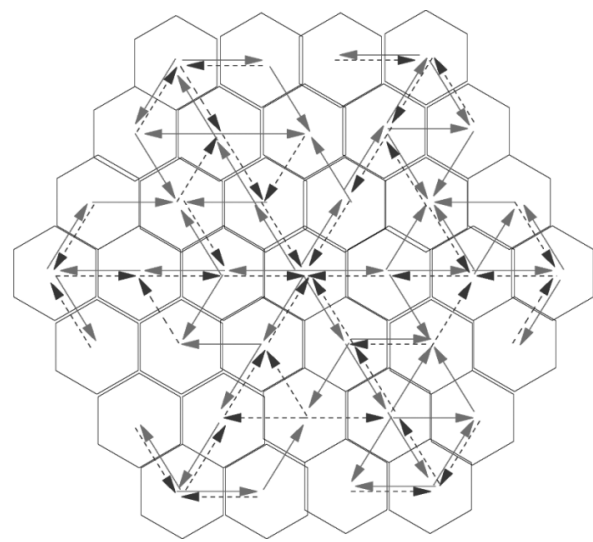

Fig. 15. Optimal network of base-stations deployed in hexagonal cells. Transmit power is $-13 \mathrm{dBm}$. Uplink rate $\lambda_{u}^{*}=0.00433$ and downlink rate $\lambda_{d}^{*}=0.0144$

are the gateways. It is assumed that each subscriber station has only uplink flow, i.e., to the gateway. Fig. 14 shows an optimal configuration when each node uses one power level $(-7.7 \mathrm{dBm})$ and one modulation scheme 1 (see Section IV). These parameters yield a transmission range of $11.4 \mathrm{~m} . \lambda^{*}$ equals 0.065 . Observe that, interestingly, almost all subscriber stations route their data only through one gateway though it is not an imposed constraint.

The next example considers a network of 36 subscriber stations deployed in hexagonal cells (of side $8 \mathrm{~m}$ ) and connected to the Internet through a base-station at the center. Each node has one uplink flow, i.e., to the base-station, and one downlink flow, i.e., from the base-station. Since the Internet traffic is asymmetric in general, the uplink flow rate is chosen to be $30 \%$ of the downlink flow. Fig. 15 shows an optimal network when each node can use one power level $(-13 \mathrm{dBm})$ and one modulation scheme of rate 1 . These parameters yield a transmission range of $8.41 \mathrm{~m}$. The red solid (resp. blue dashed) lines represent the downlink (resp. uplink) flow. The optimal uplink flow rate $\lambda_{u}^{*}$ is 0.00433 and the optimal downlink flow rate $\lambda_{d}^{*}$ is 0.0144 .

These examples demonstrate three things: (i) the optimal network is quite unlike the tree-based structures proposed for IEEE 802.16-like networks [13] (ii) mesh networks may not scale well since throughputs are fairly low even for 25 nodes (also see [23]), and (iii) our optimization/computational framework can be utilized as a tool in configuring such IEEE 802.16 based access networks.

\section{CONCLUSION}

Our work addresses the following two questions concerning the optimal throughput of fixed wireless networks: (i) what is the max-min throughput for an arbitrary set of stationary nodes and data flows? and (ii) what is the optimal network configuration to achieve this throughput? We answer these questions via a novel optimization framework, using a conflict set formulation motivated by signal decoding in the presence of noise, to model the wireless channel interference. By means of analytical results, and several numerical case-studies, we obtain important insights into the structure of optimal network configuration. In particular, we find that increasing the transmit power improves the capacity of wireless networks. This result is in contrast with power control protocols such as COMPOW [7] which recommend the use of minimum power, while maintaining connectivity. We show that the answer to the question whether power should be used to improve range or data-rate is non-trivial. We, however, provide a partial characterization which helps us to explain the results we obtain in a variety of scenarios. This is the notion of flow routes trying to minimize transmission times through resourceconstrained cliques. Related (but simplistic) notions such as minimum hop routing and minimum medium time routing [9] emerge as special cases of our characterization, and do not apply in general. Interestingly, our analytical results also capture a generalization of the classical graph inequality, namely, that the clique number of a graph is at most equal to its chromatic number.

We believe that our formulation is of direct relevance to an upcoming class of networks, which we term scheduled networks. By using static link schedules, to assign capacities to wireless links, such wireless networks, e.g., those based on IEEE 802.16, can be optimally configured and maintained.

Our work can be extended in several directions. In particular, an important assumption we have relied upon in this work, has been that of time-invariant channel gains. Although experimental studies such as [18] indicate that this is not an unreasonable assumption, it would be of great interest to be able to make the optimal network configuration "robust" to accommodate channel variations, as well as some variations in traffic demands. This would achieve the benefits of dynamic formulations, with the convenience of a static approach. Other avenues for future work include extensions to MIMO systems, problems of gateway placement, and so on.

\section{PROOFS}

We make some technical assumptions required for wellposedness of (TO).

1) $\mathcal{Z}$ is a compact uncountable set.

2) For each $P_{l}, \mathcal{Z}_{l}\left(P_{l}\right)$ is compact.

3) $\beta($.$) is a continuous function over \mathcal{Z}$.

Lemma 7.1: $Z(\mathbf{P} \mapsto \mathcal{Z}(\mathbf{P}))$ is an upper hemi-continuous correspondence.

Proof: Follows from the fact that the set $\mathcal{Z}_{l}\left(P_{l}\right)$ is increasing with $P_{l}$ and $z_{l} \in \mathcal{Z}_{l}\left(P_{l}\right)$ if and only if $G_{l l} P_{l} / N_{0} \geq \beta\left(z_{l}\right)$.

Lemma 7.2: $A((\mathbf{z}, \mathbf{P}) \mapsto \mathcal{A}(\mathbf{z}, \mathbf{P}))$ is a compact-valued upper hemi-continuous correspondence.

Proof: Compactness of $\mathcal{A}(\mathbf{z}, \mathbf{P}))$ follows from its definition. Hence, for upper hemi-continuity it is sufficient to show 
that for all sequences $(\mathbf{P}(n), \mathbf{z}(n)) \rightarrow(\mathbf{P}, \mathbf{z})$ (with $\mathbf{z}(n)$ and $\mathbf{z}$ in $\mathcal{Z}(\mathbf{P}(n))$ and $\mathcal{Z}(\mathbf{P})$ resp. $)$, and all sequences $\{\alpha(n) \in$ $\mathcal{A}(\mathbf{z}(n), \mathbf{P}(n))\}$ there is a subsequence of $\{\alpha(n)\}$ with limit in $\mathcal{A}(\mathbf{z}, \mathbf{P})$ [21]. Since $\{\alpha(n)\}$ is bounded it converges along a subsequence, say to $\alpha$. We need to show that $\alpha_{i}=0$ for $i \notin \mathcal{I}(\mathbf{z}, \mathbf{P})$. Suppose not. Then for $n \geq N$ for some $N$, $\alpha_{i}(n)>0$. This implies that $i \in \mathcal{I}(\mathbf{z}(n), \mathbf{P}(n))$ since $\alpha(n) \in$ $\mathcal{A}(\mathbf{z}(n), \mathbf{P}(n))$. Hence for some link $l \in i, \gamma_{l}(\mathbf{P})<\beta\left(z_{l}\right)$ whereas $\gamma_{l}(\mathbf{P}(n)) \geq \beta\left(z_{l}(n)\right)$. This cannot happen since $\gamma_{l}$ and $\beta$ are continuous functions of resp. parameters. Therefore, for $n$ sufficiently large $\alpha_{i}=0$ implying that an independent set cannot become non-independent suddenly at $(\mathbf{P}, \mathbf{z})$ The limiting schedule is, thus, feasible, i.e., $\alpha \in \mathcal{A}(\mathbf{z}, \mathbf{P})$.

Proof of Proposition 3.1: Let $X$ denote the constraint set of (TO). We show that $X$ is compact so that existence of an optimal solution is implied by Weierstrass theorem. Note that each $\lambda_{f}$ and hence $\lambda$ is upper-bounded by the maximum data rate on a link (obtained by non-conflicting activation for the duration of the frame with highest rate modulation under the maximum transmit power). It, thus, follows that $X$ is bounded. Lemma 7.1 and Lemma 7.2 imply upper hemi-continuity and compact-valuedness of correspondences $Z$ and $A$. This along with continuity of each constraint function implies that $X$ is closed. $X$ is, therefore, compact implying existence of an optimal solution. $\square$

Proof of Proposition 3.2: Let $i \in \mathcal{I}_{l}(\mathbf{z}, \mathbf{P})$ and let $\mathbf{v}$ denote the $\{0,1\}$ "activation vector" corresponding to $i$. Then $G_{l l} P_{l} /\left(N_{0}+\sum_{l^{\prime}} G_{l^{\prime} l} P_{l^{\prime}} v_{l^{\prime}}\right) \geq \beta\left(z_{l}\right)$. Clearly for $\xi \geq 1, G_{l l} \xi P_{l} /\left(N_{0}+\sum_{l^{\prime}} G_{l^{\prime} l} \xi P_{l^{\prime}} v_{l^{\prime}}\right)=G_{l l} P_{l} /\left(\left(N_{0} / \xi\right)+\right.$ $\left.\sum_{l^{\prime}} G_{l^{\prime} l} P_{l^{\prime}} v_{l^{\prime}}\right) \geq \beta\left(z_{l}\right)$. Therefore, $i \in \mathcal{I}_{l}(\mathbf{z}, \mathbf{P}) \Rightarrow i \in$ $\mathcal{I}_{l}(\mathbf{z}, \xi \mathbf{P})$, and $\mathcal{A}(\mathbf{z}, \mathbf{P}) \subseteq \mathcal{A}(\mathbf{z}, \xi \mathbf{P})$. Thus, if $X(\mathbf{z}, \mathbf{P})$ denotes the constraint set of (TO) for given $\mathbf{z}$ and $\mathbf{P}$, then it is clear that $X(\mathbf{z}, \mathbf{P}) \subseteq X(\mathbf{z}, \xi \mathbf{P})$.

Denote by $X$ the constraint set of (TOFINITE). Thus, $X=\left\{\sum_{f \in \mathcal{F}} \lambda_{f}\left(\sum_{r \in \mathcal{R}_{f}^{l}} \phi_{f}^{r}\right) \leq c_{l} \sum_{i \in \mathcal{I}_{l}} \alpha_{i}, l=\right.$ $1, \ldots, L, \sum_{r \in \mathcal{R}_{f}} \phi_{f}^{r}=1, \phi_{f}^{r} \geq 0, f \in \mathcal{F}, r \in \mathcal{R}_{f}, \lambda \leq$ $\left.\lambda_{f}, f=1, \ldots, M, \lambda \geq 0, \alpha \in \mathcal{A}\right\}$.

Lemma 7.3: $X$ is closed.

Proof: Let $g_{l}(\bar{\lambda}, \phi, \alpha):=\sum_{f \in \mathcal{F}} \lambda_{f}\left(\sum_{r \in \mathcal{R}_{f}^{l}} \phi_{f}^{r}\right)-c_{l}(\alpha)$. Then $g_{l}()$ is continuous. Hence $(\phi, \alpha) \rightarrow\left\{\bar{\lambda} \mid g_{l}(\bar{\lambda}, \phi, \alpha) \leq 0\right\}$ is an upper-hemicontinuous correspondence. It is compact-valued and therefore closed [21]. This means that if $(\bar{\lambda}, \phi, \alpha)_{n} \rightarrow(\bar{\lambda}, \phi, \alpha)$ with $g_{l}\left((\bar{\lambda}, \phi, \alpha)_{n}\right) \leq 0$ for each $n$, then $g_{l}((\bar{\lambda}, \phi, \alpha) \leq 0$. It is now easy to see that if $(\lambda, \bar{\lambda}, \phi, \alpha)_{n} \rightarrow(\lambda, \overline{\bar{\lambda}}, \phi, \alpha)$ with $(\lambda, \bar{\lambda}, \phi, \alpha)_{n} \in X$ for each $n,(\lambda, \bar{\lambda}, \phi, \alpha) \in X . X$ is thus closed.

Proof of Proposition 3.3: $X$ is clearly bounded; $0 \leq \lambda \leq$ $\lambda_{f} \leq \max _{l} c_{l}$. By Lemma 7.3 it is closed. Since the objective function is continuous over $X$, the proposition follows from Weierstrass theorem.

Lemma 7.4: $\Theta(\phi)$ is compact and convex.

Proof: Compactness is easy to see. Convexity follows by noting that it is obtained as the intersection of convex sets- half-planes $\left(\sum_{f \in \mathcal{F}} \lambda_{f}\left(\sum_{r \in \mathcal{R}_{f}^{l}} \phi_{f}^{r}\right) \leq c_{l} \sum_{i \in \mathcal{I}_{l}} \alpha_{i} l=\right.$ $\left.1, \ldots, L, \lambda \leq \lambda_{f} f=1, \ldots, M, \lambda \geq 0\right)$ and the convex set $\mathcal{A} . \square$

Lemma 7.5: $\Theta$ is a continuous correspondence.

Lemma 7.6: Let $g(x, r)$ be continuous function over $X \times R$. If for every $r$, there exists $x \in X$ such that $g(x, r)<0$ then the correspondence $r \rightarrow\{x \mid g(x, r) \leq 0\}$ is continuous.
Proof of Lemma 7.5: For each $\phi \in \Phi$, there exist $(\bar{\lambda}, \alpha)$ such that $\sum_{f \in \mathcal{F}} \lambda_{f}\left(\sum_{r \in \mathcal{R}_{f}^{l}} \phi_{f}^{r}\right)<c_{l}(\alpha)$ (choose $\alpha \in \mathcal{A}$ such that $c_{l}(\alpha)>0$ for each $l$ and $\lambda_{f}=0$ for each $f$ ). Therefore, $\phi \rightarrow\left\{(\lambda, \bar{\lambda}, \alpha) \mid \sum_{f \in \mathcal{F}} \lambda_{f}\left(\sum_{r \in \mathcal{R}_{f}^{l}} \phi_{f}^{r}\right) \leq c_{l}(\alpha)\right\}$ is continuous by Lemma 7.6. Since $\Theta$ is restriction of this over $\left\{\mathcal{A}, \lambda_{f} \geq\right.$ $\lambda \geq 0\}$, it is continuous.

Proof of Proposition 3.4:

1) Follows from Lemma 7.4.

2) Follows from Lemma 7.5, the Maximum theorem [21] and the fact that $\lambda^{*}(\phi)$ is continuous over compact convex set $\Phi$.

3) The parameterized version of (2) has linear constraint functions and an abstract set constraint $(\mathcal{A})$. A feasible solution exists in the interior of the abstract set constraint (use say clique feasibility constraint). Proposition follows from [20].

Proof of Proposition 3.5: $\lambda_{f}^{*}(\phi)_{f \in \mathcal{F}}$ are optimal, hence feasible flow rate in the parameterized problem. This implies that $\sum_{l \in c}\left(\sum_{f \in \mathcal{F}} \lambda_{f}^{*}(\phi)\left(\sum_{r \in \mathcal{R}_{f}^{l}} \phi_{f}^{r}\right) / c_{l}\right) \leq 1$ for each $c \in \mathcal{C}$. Recall that this is a necessary condition for schedulability in terms of clique feasibility [19]. Since $\lambda^{*}(\phi) \leq \lambda_{f}^{*}(\phi)$ for each $f \in \mathcal{F}$, it follows that

$$
\lambda^{*}(\phi) \leq \frac{1}{\max _{c} w_{c}(\phi)}
$$

On the other hand,

$$
\sum_{l \in c} \frac{\sum_{f \in \mathcal{F}} \lambda_{f}(\phi)\left(\sum_{r \in \mathcal{R}_{f}^{l}} \phi_{f}^{r}\right)}{c_{l}} \leq \kappa
$$

implies there exist $(\lambda, \bar{\lambda}, \alpha) \in \Theta(\phi)$ realizing flow rates $\left(\lambda_{f}\right)_{f \in \mathcal{F}}$. This is, therefore, a sufficient condition for feasible flow rates under given routing variables. Clearly over all flow rates satisfying (13) the optimal solution of the parameterized problem is $\hat{\lambda}(\phi):=\kappa / \max _{c} w_{c}(\phi)$. Since $\lambda^{*}(\phi) \geq \hat{\lambda}(\phi)$, it follows that

$$
\frac{\kappa}{\max _{c} w_{c}(\phi)} \leq \lambda^{*}(\phi) \leq \frac{1}{\max _{c} w_{c}(\phi)}
$$

Proposition now follows from Proposition 3.4 (1).

Proof of Proposition 3.6: Right inequality is straightforward (see Proposition 3.4 (2)). For the left inequality note that

$$
\begin{aligned}
\lambda^{*}(\hat{\phi}) & \geq \frac{\kappa}{\max _{c} w_{c}(\hat{\phi})} \\
& =\frac{\kappa}{\min _{\phi} \max _{c} w_{c}(\phi)} \\
& \geq \kappa \lambda^{*}
\end{aligned}
$$

(14) follows from the clique sufficiency condition (see Proof of Proposition 3.5), (15) from the definition of $\hat{\phi}$ as the "min-max routing" and (16) from the left inequality in Proposition 3.5. $\square$

Proof of Proposition 4.2: We show this by using a packing argument to place the transmitters of links, as closely as possible, such that the links still form an independent set. Consider two links, say $l$ and $m$. Without loss of generality, let the transmitter of link $m, m_{o}$, use higher transmit power. Let us denote the transmitter-receiver separation of link $l$ by $x$, and the distance of $m_{o}$ from $l_{d}$ by $y$. Now, under the assumptions A1-A3, the minimum value of $y$ for a given $x$, so that link $m$ does not interfere with link $l$ is bounded below by $x \beta_{\min }^{1 / \eta}$. This can be derived as follows. Use $P$ to denote the transmit power of link $m$. Then the signal 
strength of link $l$ is upper bounded by $P\left(x / d_{0}\right)^{-\eta}$, and the interference perceived by link $l$ is lower bounded by $P\left(y / d_{0}\right)^{-\eta}$. This gives an upper bound on the SINR of link $l$, and therefore a lower bound of $y$ so that $l$ does not encounter a packet decoding failure. Now, by the triangle inequality,

$$
\begin{aligned}
\left|m_{o}-l_{o}\right| & \geq\left|m_{o}-l_{d}\right|-\left|l_{d}-l_{o}\right| \\
& =y-x \geq x\left(\beta_{\min }^{1 / \eta}-1\right) \\
& \geq \max \left\{d_{\min }, d_{\min }\left(\beta_{\min }^{1 / \eta}-1\right)\right\}
\end{aligned}
$$

where the last inequality follow from the fact that nodes are separated by a minimum distance $d_{\min }$. Thus, for the links $l$ and $m$ to be independent, the distance between their transmitters has to exceed $\max \left\{d_{\min }, d_{\min }\left(\beta_{\min }^{1 / \eta}-1\right)\right\}$. This is equivalent to embedding each transmitter at the center of an exclusion disc of radius $(1 / 2) \max \left\{d_{\min }, d_{\min }\left(\beta_{\min }^{1 / \eta}-1\right)\right\}$, and requiring discs to be non-intersecting, in order for the corresponding links to be independent. Since the network is confined to a region of area $L^{2}$, the maximum size of an independent set is upper bounded by,

$$
B=\frac{4 L^{2}}{\pi\left(\max \left\{d_{\min }, d_{\min }\left(\beta_{\min }^{1 / \eta}-1\right)\right\}\right)^{2}}
$$

\section{ACKNOWLEDGMENT}

The authors would like to thank S. Muthaiah, University of Waterloo, for help with some numerical results, and the reviewers for their helpful comments.

\section{REFERENCES}

[1] P. Gupta and P. R. Kumar, "The capacity of wireless networks," IEEE Trans. Inf. Theory, vol. 46, no. 2, pp. 388-404, Mar. 2000.

[2] V. Mhatre and C. Rosenberg, "The impact of link layer model on the capacity of random ad hoc network," in Proc. 2006 ISIT, Jul. 2006.

[3] L.-L. Xie and P. R. Kumar, "A network information theory for wireless communication: Scaling laws and optimal operation," IEEE Trans. Inf. Theory, vol. 50, no. 5, pp. 748-767, May 2004.

[4] J. Proakis, Digital Communications. New York: McGraw-Hill, 2000

[5] A. Iyer, C. Rosenberg, and A. Karnik, "What is the right model for wireless channel interference?," in Proc. QShine, 2006.

[6] A. Karnik and A. Kumar, "Distributed optimal self-organisation in a class of wireless sensor networks," in Proc. IEEE INFOCOM, 2004, pp. 536-547.

[7] S. Narayanaswamy, V. Kawadia, R. S. Sreenivas, and P. R. Kumar, "Power control in ad hoc networks: Theory, architecture, algorithm and implementation of the COMPOW protocol," in Proc. Eur. Wireless Conf., 2002.

[8] A. Behzad and I. Rubin, "High transmission power increases the capacity of ad hoc wireless networks," IEEE Trans. Wireless Commun., vol. 5, no. 1, pp. 156-165, Jan. 2006.

[9] B. Awerbuch, D. Holmer, and H. Rubens, "The medium time metric: High throughput route selection in multi-rate ad hoc wireless networks," ACM Mobile Networks and Applications, vol. 11, no. 2, pp. 253-266, Apr. 2006.

[10] I. F. Akyildiz, X. Wang, and W. Wang, "Wireless mesh networks: A survey," Computer Networks, Elsevier Science, vol. 47, pp. 445-487, Mar. 2005.

[11] K. Jain, J. Padhye, V. N. Padmanabhan, and L. Qiu, "Impact of interference on multi-hop wireless network performance," in ACM/IEEE MobiCom, 2003.

[12] P. Stuedi and G. Alonso, "Computing throughput capacity for realistic wireless multihop networks," in Proc. ACM MSWiM'06, 2006.

[13] H. Viswanathan and S. Mukherjee, "Throughput-range tradeoff of wireless mesh backhaul networks," IEEE J. Sel. Areas Commun., vol. 24, no. 3, pp. 593-602, Mar. 2006.

[14] R. L. Cruz and A. V. Santhanam, "Optimal routing, link scheduling and power control in multihop wireless networks," in Proc. IEEE INFOCOM, 2003, pp. 702-711.
[15] M. J. Neely, E. Modiano, and C. E. Rohrs, "Dynamic power allocation and routing for time-varying wireless networks," IEEE J. Sel. Areas Commun., vol. 23, no. 1, pp. 89-103, Jan. 2005.

[16] L. Tassiulas and A. Ephremides, "Stability properties of constrained queueing systems and scheduling policies for maximum throughput in multihop radio networks," IEEE Trans. Automat. Contr., vol. 37, no. 12, pp. 1936-1948, Dec. 1992.

[17] R. Gallager, "A minimum delay routing algorithm using distributed computation," IEEE Trans. Commun., vol. COM-25, no. 1, pp. 73-85, Jan. 1977.

[18] V. Erceg, L. Greenstein, Y. Tjandra, S. Parkoff, A. Gupta, B. Kulic, A. Julius, and R. Bianchi, "An empirically-based path loss model for wireless channels in suburban environments," IEEE J. Sel. Areas Commun., vol. 17, no. 7, pp. 1205-1211, Jul. 1999.

[19] A. Bar-Noy, A. Mayer, B. Schieber, and M. Sudan, "Guaranteeing fair service to persistent dependent tasks," SIAM J. Computing, vol. 27, no. 4, pp. 1168-1189, Aug. 1998.

[20] D. Bertsekas, Nonlinear Programming. Nashua, NH: Athena Scientific, 1995.

[21] K. Border, Fixed Point Theorems With Applications to Economics and Game Theory. Cambridge, U.K.: Cambridge Univ. Press, 1985.

[22] R. Gupta, J. Musacchio, and J. Walrand, "Sufficient rate constraints for QoS Flows in ad hoc networks," Ad Hoc Networks J., to be published.

[23] S. Muthaiah, A. Iyer, A. Karnik, and C. Rosenberg, "Design of high throughput scheduled wireless mesh networks: Smart antennas," in Proc. IEEE Globecom, 2007.

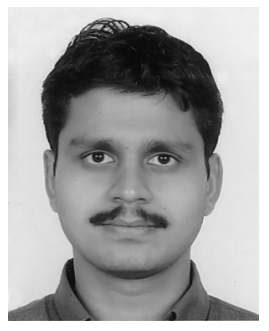

Aditya Karnik received the B.E. degree in electronics and telecommunications from the University of Pune, Pune, India, and the M.E. and Ph.D degrees, both in electrical communication engineering, from the Indian Institute of Science, Bangalore, India. During his Ph.D degree work, he was a recipient of the IBM Research Fellowship.

$\mathrm{He}$ is currently with the Manufacturing Enterprise Modeling Group, General Motors India Science Laboratory, Bangalore. His research interests are in control and optimization theory in general, and its application to communication and manufacturing systems in particular.

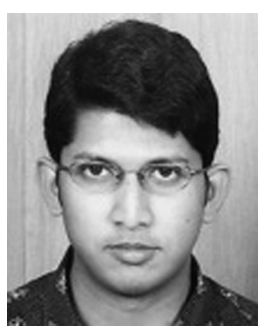

Aravind Iyer received the B.Tech. and M.Tech. degrees in electrical engineering from the Indian Institute of Technology (IIT), Bombay, in August 2002, and the Ph.D. degree from the School of Electrical and Computer Engineering, Purdue University, West Lafayette, IN, in May 2007.

$\mathrm{He}$ is currently with the Vehicular Communication and Information Management Group, General Motors India Science Laboratory, Bangalore. His research interests include the design, modeling, and optimization of multi-hop wireless networks such as sensor networks, ad hoc networks, and wireless mesh networks.

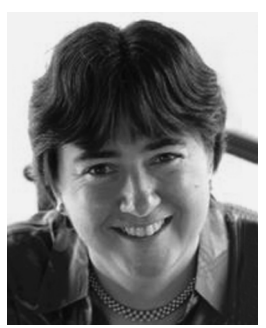

Catherine Rosenberg (SM'95) was born in France. She received the Diplôme d'Ingénieur degree in electrical engineering from the Ecole Nationale Supérieure des Télécommunications de Bretagne in 1983, the Doctorat en Sciences degree in computer science from the University of Paris, Orsay, in 1986, and in the M.S. degree in computer science from the University of California, Los Angeles, in 1984.

She has worked in several countries including the USA, U.K., Canada, France, and India. In particular, she worked for Nortel Networks in the U.K., AT\&T Bell Laboratories in the USA, and Alcatel in France, and taught at Purdue University, West Lafayette, IN, and Ecole Polytechnique of Montreal, Montreal, QC, Canada. In 2004, she joined the University of Waterloo, Canada, where she holds a University Research Chair. In 2007, she was elected Member at Large of the Board of Governors of the Communications Society (ComSoc) of the IEEE for a two-year term and was invited to join the Scientific Board of France-Telecom for a three-year term. Her research interests are broadly in networking with currently an emphasis in wireless networking and in traffic engineering (quality of service, network design, and routing). She has authored over 100 papers and has been awarded eight U.S. patents. 